\title{
Increasing effect of ceramides in skin by topical application of sphingosine
}

\author{
Koji Nomoto ${ }^{1}$, Fumie Hashimoto ${ }^{2}$, Kenji Sugibayashi ${ }^{2}$ and Yoshihiro Tokudome To $^{2 *}$ \\ ${ }^{1}$ Doctoral student, Faculty of Pharmaceutical Sciences, Josai University, 1-1 Keyakidai, Sakado, Saitama, 350-0295, Japan \\ ${ }^{2}$ Professor, Faculty of Pharmaceutical Sciences, Josai University, 1-1 Keyakidai, Sakado, Saitama, 350-0295, Japan
}

\begin{abstract}
Introduction and objective: Ceramidase metabolizes ceramide and generates sphingoid and fatty acid. Alkaline ceramidase has been involved in reduction of the ceramide level in atopic dermatitis or in aged dry skin. We hypothesized that the ceramide content can be increased by inhibition of the ceramidase activity. In this study, we aimed to examine the effect of alkaline ceramidase inhibitor on the amount of the ceramide in mice skin and in three-dimensional cultured epidermis model.

Material and methods: 4-Nitrobenzo-2-oxa-1,3-diazole-labeled ceramide was used as a substrate and was incubated with skin homogenate at $37^{\circ} \mathrm{C}$ for 1 or 10 h. Fluorescent-labeled fatty acid, enzymatic reaction product was detected by fluorescence high performance liquid chromatography to determine the alkaline ceramidase activity. The ceramide content was quantitatively analyzed by high performance thin layer chromatography.

Results: 50\% Inhibitory concentration of sphingosine for alkaline ceramidase in mice skin and three-dimensional cultured epidermis model was $>70$-fold lower than the corresponding oleoylethanolamide. Ceramide [AS] and ceramide [AP] contents of sphingosine-applied group of mice stratum corneum were significantly higher than in the normal group. Compared with the normal group, ceramide [NDS] in three-dimensional cultured epidermis model was also significantly increased in sphingosine-applied group.

Conclusion: This study demonstrated that sphingosine inhibited alkaline ceramidase in mice skin and three-dimensional cultured epidermis model and ceramide contents was increased by application of sphingosine. These results suggest that sphingosine was beneficial compound to increase skin ceramide, though sphingosine may also be affected by another pathway.
\end{abstract}

\section{Introduction}

Ceramide (CER) in the stratum corneum (SC) has an important role in water retention as well as skin barrier function. Ceramidase (CDase) hydrolyzes the $\mathrm{N}$-acyl linkage of CER and converts CER to sphingoid and fatty acid (FA). Houben and Lin reported that the human epidermis had acidic CDase (aCDase) and alkaline CDase (alkCDase) activities [1,2]. It was also demonstrated that alkCDase 1 mRNA expression level in human epidermis was higher than other tissues; total alkCDase and aCDase were activated, and mRNA expression of alkCDase 1 and aCDase was increased by differentiation of keratinocyte [3].

Skin barrier function is altered by skin conditions such as aging and atopic dermatitis $(\mathrm{AD})$. Imokawa and Ghadially revealed a decrease in the CER content in SC in aged human and hairless mice [4,5]. Jin et al. demonstrated that the alkCDase activity ( $\mathrm{pH} 9.0$ ) was elevated in aged human SC compared with young [6]. AD patients showed high transepidermal water loss (TEWL) and low total CER content in SC [4]. Sphingomyelin deacylase and glucosylceramide deacylase were closely related to AD [7]. However, Okino found that alkCDase was secreted from Pseudomonas aeruginosa in skin of $\mathrm{AD}$ patients [8,9] and considered that it subsequently decreased the CER content [10]. But little has been reported on regulation of the CER content in SC by inhibition of the CDase.

We then hypothesized that CDase inhibitor may increase the CER content in SC. Yada and Mao reported that sphingosine (SPH) inhibited mouse alkaline ceramidase (maCER1) and guinea pig epidermal alkCDase activity [11,12]. In this study, we used hairless mice and three-dimensional cultured epidermis model, and reported the effects of SPH on the alkCDase activity and the CER content.

\section{Material and methods}

\section{Animals and three-dimensional cultured epidermis model}

Male HOS:HR-1 mice, 4 and 7 week sold, were purchased from Japan SLC, Inc. (Hamamatsu, Shizuoka, Japan) and were kept under a 12 -h light and 12 -h dark cycle in a conventional animal room maintained at $22 \pm 2{ }^{\circ} \mathrm{C} ; 55 \% \pm 5 \%$ and free access to food and water. All procedures were approved by the Ethics Committee of Josai University (Sakado, Saitama, Japan). Six-day cultured LabCyte EPI-MODEL was obtained from Japan Tissue Engineering Co., Ltd. (Gamagori, Aichi, Japan).

\section{Materials}

Fluorescent-labeled 4-nitrobenzo-2-oxa-1,3-diazole (NBD) group FAs ( $\mathrm{C}_{6}$-NBD-FA and $\mathrm{C}_{12}$-NBD-FA) were purchased from Molecular Probes (Eugene, OR, USA) and Invitrogen (Waltham, MA, USA). $\mathrm{C}_{12}{ }^{-}$

Correspondence to: Yoshihiro Tokudome, Professor, Faculty of Pharmaceutical Sciences, Josai University, 1-1 Keyakidai, Sakado, Saitama, 350-0295, Japan, Tel: +81-49-271-8140; E-mail: tokudome@josai.ac.jp

Keywords: Ceramidase inhibitor, Ceramide, Skin, Sphingosine, Stratum corneum

Received: May 28, 2015; Accepted: July 14, 2015; Published: July 17, 2015 
NBD-CER was purchased from Cayman Chemical Company (Ann Arbor, MI, USA). Oleoylethanolamide (OEA) was obtained from Enzo Biochem, Inc. (New York, NY, USA). D-Erythro-sphingosine was obtained from Wako Pure Chemical Industries (Osaka, Japan). CER standard for quantitative determination was obtained from Matreya, LLC (Pleasant Gap, PA, USA), Evonik Industries (Essen, North Rhine-Westphalia, Germany), and Takasago International Corporation (Tokyo, Japan). Dipalmitoylphosphatidylcholine (DPPC) and dipalmitoylphosphatidylglycerol (DPPG) were kindly donated by Nippon Fine Chemical (Osaka, Japan). Other reagents were obtained from Wako Pure Chemical Industries, Daiichi Sankyo Co., Ltd. (Tokyo, Japan), Tokyo Chemical Industry Co., Ltd. (Tokyo, Japan), Alfa Aesar (Heysham, Lancashire, UK) and Sigma-Aldrich Co., LLC. (St. Louis, MO, USA).

\section{Preparation of homogenate}

Mice were killed by cervical dislocation and dorsal skin was obtained. Subcutaneous tissue was removed by scissors. After weighing, the skin was minced using scissors for $3 \mathrm{~min}$ on ice and was homogenized in $0.25 \mathrm{M}$ sucrose, supplemented with $1 \%$ Triton X-100 using Polytron PT 1200 E homogenizer (Kinematica AG, Luzernerstrasse, Lucern, Switzerland) at $25,000 \mathrm{rpm}$ for $1 \mathrm{~min}$ on ice. The homogenate was centrifuged at $500 \times \mathrm{g}$ at $4^{\circ} \mathrm{C}$ for $1 \mathrm{~min}$ and the supernatant was stored at $-30^{\circ} \mathrm{C}$ until use. Three-dimensional cultured epidermal models were collected from transwell by spatula and homogenized, as described above. Protein concentration was determined by a modified Lowry method.

\section{Measurement of the alkCDase and aCDaseactivity}

The CDase activity was measured by the method of Houben et al., with some modifications [1]. NBD-C $\mathrm{C}_{12}$-CER was used as substrate of CDase. An appropriate amount of NBD-C ${ }_{12}$-CER, with final concentration of $250 \mu \mathrm{M}$, was dissolved in methanol and allowed to dry under nitrogen gas. The substrate was redissolved by sonication $\left(50^{\circ} \mathrm{C}, 10 \mathrm{~min}\right)$ in $12.5 \mu \mathrm{l}$ of detergent mixture containing $40 \mathrm{mg} / \mathrm{ml}$ Triton X-100, $16 \mathrm{mg} / \mathrm{ml}$ Tween 80 , and $25 \mu \mathrm{l}$ of $250 \mathrm{mM}$ Tris- $\mathrm{HCl}$ buffer (for alkCDase, $\mathrm{pH}$ 8.5) or acetate buffer (for aCDase, $\mathrm{pH}$ 4.2). Mice skin or three-dimensional cultured epidermis model homogenate was preincubated at $37^{\circ} \mathrm{C}$ for $2 \mathrm{~min}$. Enzymatic reactions were initiated by the addition of $12.5 \mu \mathrm{l}$ of $60 \mathrm{ng}$ protein $/ \mathrm{ml}$ homogenate, and the final concentration was $15 \mathrm{ng}$ protein $/ \mathrm{ml}$ to the substrate solution at $37^{\circ} \mathrm{C}$. Incubation time was $1 \mathrm{~h}$ for mice skin or $10 \mathrm{~h}$ for three-dimensional cultured epidermis model. The reaction was stopped by addition of 300 $\mu \mathrm{l}$ of chloroform/methanol (2:1) that contained $0.26 \mu \mathrm{M}$ NBD-C ${ }_{6}-\mathrm{FA}$ as internal standard for HPLC analysis. The reaction mixture was dried under nitrogen gas and dissolved in $100 \mu \mathrm{l}$ of methanol. HPLC system (SHIMADZU Prominence HPLC system, SHIMADZU Corp., Kyoto, Kyoto, Japan), equipped with a reversed phase L-Column 2 ODS (5 $\mu \mathrm{m}, 2.1 \times 150 \mathrm{~mm}$; Chemicals Evaluation and Research Institute, Tokyo, Japan), and a fluorescence detector (RF-10AXL, SHIMADZU) set to excitation and emission wavelengths of 460 and $534 \mathrm{~nm}$, respectively, were used to determine NBD- $\mathrm{C}_{12}$-FA in the enzymatic reaction sample. Column was maintained at $30^{\circ} \mathrm{C}$. The mobile phases were $0.1 \%$ formic acid in acetonitrile (A) and $0.1 \%$ formic acid in water (B). A binary mobile phase at a flow rate of $0.4 \mathrm{ml} / \mathrm{min}$ was used under liner gradient conditions as follows: $0 \mathrm{~min}, 30 \% \mathrm{~A} ; 0-5 \mathrm{~min}, 30 \%-100 \% \mathrm{~A} ; 5-14.5$ min, $100 \%$ A; $14.5-14.51 \mathrm{~min}, 30 \%$ A; $14.51-30 \mathrm{~min}, 30 \%$ A. Standard curve of peak height ratio (NBD-C $-\mathrm{FA}$ vs. internal standard) was used to determine NBD- $\mathrm{C}_{12}-\mathrm{FA}$ that was formed during the enzymatic reaction. To correct the CDase activity values, the NBD- $\mathrm{C}_{12}-\mathrm{FA}$ content in an incubated reaction mixture without protein was subtracted from that in a reaction mixture with protein. To assess inhibitory effect, SPH dissolved in methanol was added to the substrate and dried. The inhibitory activity was measured as described above. OEA, which has the inhibitory activity for alkCDase and aCDase [1], was used as a positive control of CDase inhibitor.

\section{Application of SPH and extract of SC lipids in mice}

SPH and OEA were dissolved in propyleneglycol/ethanol (7:3; concentration, $40 \mathrm{mM}$ ) [13]. Samples of $50 \mu \mathrm{l}$ were applied twice a day for three days to the dorsal skin (area, 5-6 $\mathrm{cm}^{2}$ ) of mice. Control group was exposed to propyleneglycol/ethanol $(7: 3)$. SC sheet was collected by stripping the dorsal skin area with applied sample with cyanoacrylate resin and was stored frozen at $-80^{\circ} \mathrm{C}$ until use. Lipids were extracted by the method of Imokawa et al. [4]. SC sheets were immersed in hexane/ethanol (95:5) and sonicated for $20 \mathrm{~min}$. Solutions of extracted lipids were filtered (MILLEX-GV Syringe Driven Filter Unit, Merck Millipore, MA, USA) and transferred to test tubes. Organic solvent was dried under nitrogen gas stream at $50^{\circ} \mathrm{C}$. Lipid in test tube was dissolved in chloroform/methanol (2:1) and sonicated at $37^{\circ} \mathrm{C}$ for $5 \mathrm{~min}$. Extract was transferred to a $1.5 \mathrm{ml}$ tube and dried. SC lipid samples were stored frozen at $-80^{\circ} \mathrm{C}$ until use.

\section{Application of SPH and extraction of epidermal lipids for three-dimensional cultured epidermis model}

Liposome constructed SPH/DPPC/DPPG (molar ratio 4:4:1) was prepared by the thin layer method previously described [14]. $\mathrm{SPH}$ concentration in a liposome was $10 \mathrm{mM}$. Three-dimensional cultured epidermis model was cultured at $37^{\circ} \mathrm{C}$ under $5 \% \mathrm{CO}_{2}$. A 50 $\mu \mathrm{l}$ solution of liposome containing SPH was applied to the SC side of three-dimensional cultured epidermis model for 6 days and was changed every 2 days. Medium was changed every day for 6 days and was supplied with $25 \mu \mathrm{g} / \mathrm{ml}$ ascorbic acid on the seventh day. After $24 \mathrm{~h}$, three-dimensional cultured epidermis model was collected from transwell by scalpel. Control group was applied with SPH-free liposome (DPPC/DPPG, 4:1). Three-dimensional cultured epidermal samples were immersed in chloroform/methanol (2:1) and sonicated by SONIFIRE 250 (Branson, Danbury, CT, USA) on ice. Lipid collection was conducted as described above.

\section{Quantification of the CER}

The CER was quantitated by the method of Imokawa et al. [4]. Extracted lipids were redissolved in chloroform/methanol (2:1) and 10 $\mu \mathrm{l}$ of extract was spotted by capillary on the high-performance thinlayer chromatography (HPTLC) Silica gel 60, measuring $20 \times 10 \mathrm{~cm}$ (Merck Millipore). The CER was isolated by chloroform/methanol/ acetic acid (190:9:1 to $9 \mathrm{~cm})$ and was developed twice. The HPTLC plate was sprayed with coloring reagent (10\% cupric sulfate, $8 \%$ phosphoric acid) and heated at $180^{\circ} \mathrm{C}$ for $10 \mathrm{~min}$ by TLC Plate Heater III (CAMAG, Muttenz, Switzerland). HPTLC plate was imaged by LAS1000-plus (Fujifilm Corporation, Tokyo, Japan) and the CER was quantitated from band density by Multi Gauge Version 3.0 (Fujifilm Corporation).

\section{Data analysis}

Data were expressed as the mean \pm S.D. $(n=4)$ and compared by Turkey's test, Dunnett's test, or Student's t-test using SAS version 9.2 (SAS Institute ltd., Cary, NC, USA). 


\section{Results}

\section{The CDase activity in mice skin}

Activity of alkCDase in the presence of $10 \mathrm{mM}$ SPH on mice skin homogenate was measured. Compared with the normal group, the alkCDase activity was significantly reduced by $46 \%$ in OEA (positive control) group $(p<0.05)$ and $88 \%$ in SPH group $(p<0.001)$. The alkCDase activity in the SPH group was significantly decreased by $78 \%$ $(p<0.05)$ relative to the OEA group (Figure 1A). Similarly, the aCDase activity in the SPH group had a tendency to be lower than that in normal group, in spite of no significant (Figure 1B). Subsequently, the inhibitory activity of SPH on the alkCDase was determined. SPH and OEA dose-dependently inhibited the alkCDase activity (Figure 2 ). The $\mathrm{IC}_{50}$ value of SPH was significantly lower than that of OEA $(p<0.001)$ (Table 1).

\section{The CER content in mice SC}

The CER content in mice SC was measured after application of 40 mM SPH or OEA. CER [NS] and CER [NP] contents were not changed
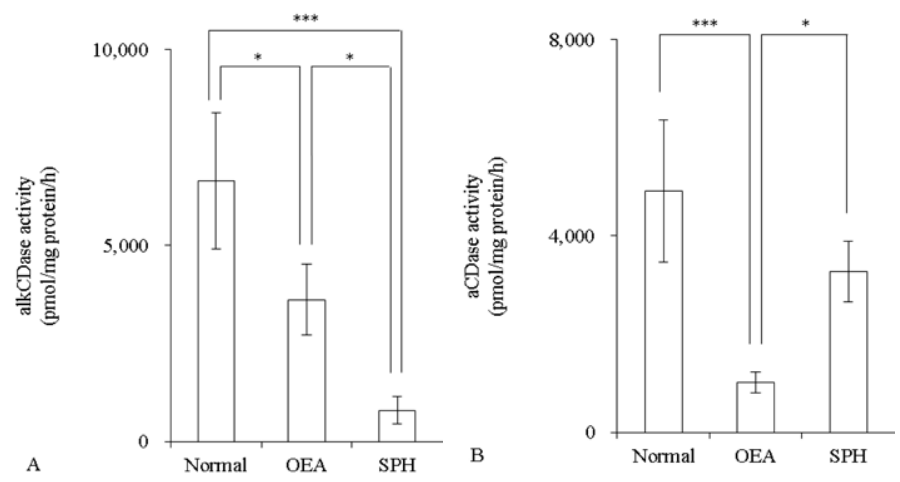

Figure 1: Effect of SPH on the CDase activity in hairless mice skin homogenate $(n=4)$ OEA was used as positive control. OEA and SPH concentrations were $10 \mathrm{mM}$. The alkCDase activity at $\mathrm{pH} 8.5$ (A) and the aCDase activity at $\mathrm{pH} 4.2$ (B). Values are expressed as the mean \pm S.D. ${ }^{*} p<0.05,{ }^{* * *} p<0.001$, as determined by Turkey's test.
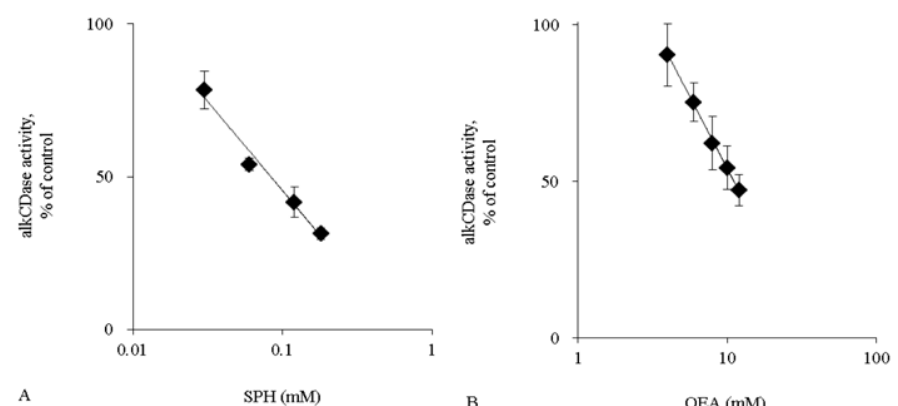

Figure 2: The alkCDase activity in hairless mice skin homogenate in the presence of alkCDase inhibitors at various concentrations $(n=4)$

OEA was used as positive control. The alkCDase activity of control was measured in an alkCDase inhibitor-free condition. SPH (0.03-0.18 mM) (A) and OEA (4-12 mM) (B). Values are expressed as the mean \pm S.D.

Table 1. $\mathrm{IC}_{50}$ values of alkCDase inhibitors on the hairless mice skin homogenate.

\begin{tabular}{|c|c|}
\hline Inhibitor & $\mathbf{I C}_{\mathbf{5 0}}$ value (mM) \\
\hline OEA & $11.1 \pm 1.59$ \\
\hline SPH & $0.09 \pm 0.01 * * *$ \\
\hline
\end{tabular}

Values are expressed as the mean \pm S.D. $(\mathrm{n}=4) . * * * p<0.001$, determined by Student's t-test.
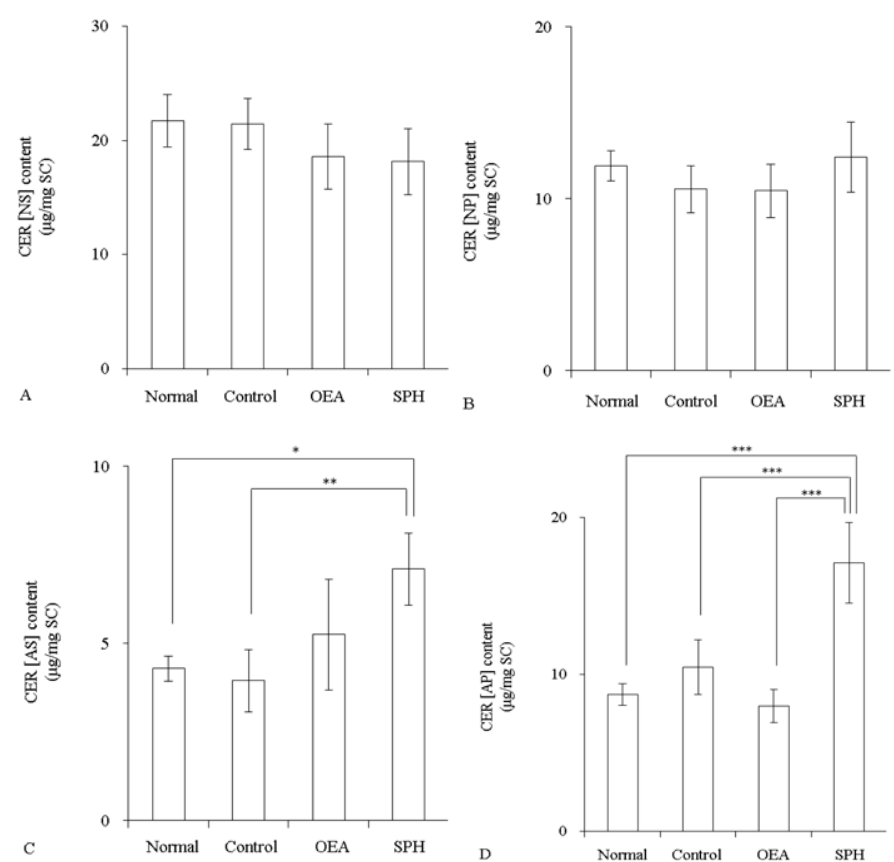

Figure 3: The CER content in SC of hairless mice after topical application of OEA or SPH $(\mathrm{n}=4)$

OEA and SPH concentrations were $40 \mathrm{mM}$. Control group was applied with propyleneglycol/ ethanol (7:3). Content of CER [NS] (A), CER [NP] (B), CER [AS] (C), and CER [AP] (D). Values are expressed as the mean \pm S.D. ${ }^{*} p<0.05,{ }^{*} p<0.01,{ }^{* * *} p<0.001$, determined by Turkey's test.

by the application of OEA or SPH (Figure $3 \mathrm{~A}$ and $3 \mathrm{~B}$ ). In contrast, CER [AS] in the SPH group was significantly increased relatively to the normal group $(p<0.05)$ (Figure 3C). CER [AP] content was also significantly increased by application of $\mathrm{SPH}$ when compared with the normal group $(p<0.001)$ (Figure 3D).

\section{The alkCDase activity in three-dimensional cultured epidermis model}

Activity of alkCDase in the presence of $10 \mathrm{mM}$ SPH on the threedimensional cultured epidermis model homogenate was measured. Compared with the normal group, the alkCDase activity was significantly decreased by $56 \%$ in OEA group and $83 \%$ in SPH group $(p<0.001)$. The alkCDase activity in the SPH group was significantly reduced by $62 \%(p<0.01)$ relatively to the OEA group (Figure 4$)$. SPH and OEA inhibited alkCDase in a dose-dependent manner (Figure 5). In agreement with the animal experiment, the $\mathrm{IC}_{50}$ value of $\mathrm{SPH}$ for alkCDase was significantly reduced compared with that of OEA $(p<0.01)$ (Table 2).

\section{The CER content in three-dimensional cultured epidermis model}

The CER content was measured after application of liposome containing $10 \mathrm{mM}$ SPH. SPH did not change the amounts of CER [NS], CER [NP], CER [AS], and CER [AP] (Figure 6A-6D). However, CER [NDS] was significantly increased in the SPH group in comparison with the normal group $(p<0.001)$ (Figure 6E).

\section{Discussion}

First, the inhibitory activity of SPH for alkCDase was assessed by comparison with OEA (positive control). The $\mathrm{IC}_{50}$ values of SPH were lower than OEA (Tables 1 and 2). Mao et al. reported that the $\mathrm{IC}_{50}$ 


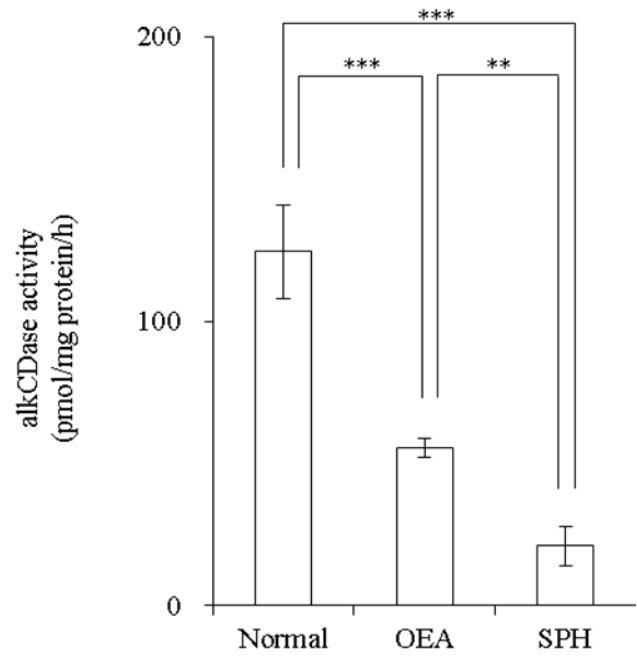

Figure 4: Effect of SPH on the alkCDase activity in three-dimensional cultured epidermis model homogenate $(\mathrm{n}=4)$

OEA was used as a positive control. OEA and SPH concentrations were $10 \mathrm{mM}$. Values are expressed as the mean \pm S.D. ${ }^{* *} p<0.01,{ }^{* * *} p<0.001$, determined by Turkey's test.
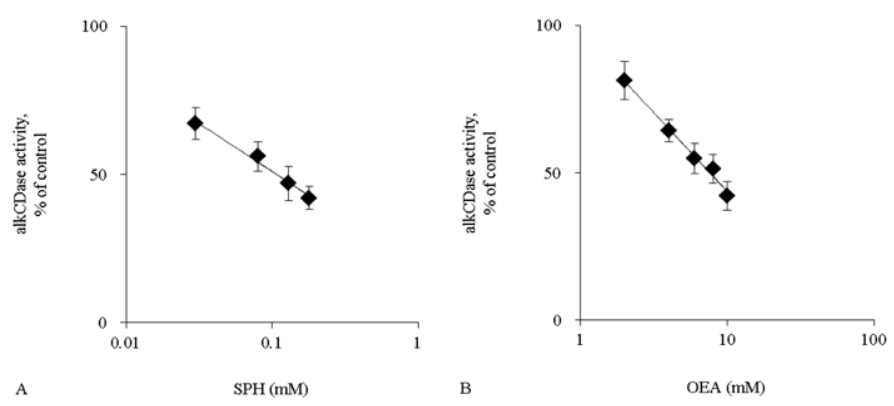

Figure 5: The alkCDase activity in three-dimensional cultured epidermis model homogenate in the presence of alkCDase inhibitors at various concentrations $(n=4)$

OEA was used as a positive control. The alkCDase activity of control was measured in an alkCDase inhibitor-free condition. SPH (0.03-0.18 mM) (A) and OEA (2-10 mM) (B). Values are expressed as the mean \pm S.D.

Table 2. $\mathrm{IC}_{50}$ values of alkCDase inhibitors in the three-dimensional cultured epidermis model homogenate.

\begin{tabular}{|c|c|}
\hline Inhibitor & IC $_{\mathbf{5 0}}$ value (mM) \\
\hline OEA & $7.77 \pm 1.21$ \\
\hline SPH & $0.11 \pm 0.03 * *$ \\
\hline
\end{tabular}

Values are expressed as the mean \pm S.D. $(\mathrm{n}=4) .{ }^{*} p<0.01$, determined by Student's t-test.

value of SPH for maCER1 ( $\mathrm{pH}$ 8.0) was $-0.08 \mathrm{mM}$ [12]. The mice skin $\mathrm{IC}_{50}$ values of SPH reported in this paper were similar to the previous study. In addition, the $\mathrm{IC}_{50}$ values of SPH and OEA were similar between mice skin and three-dimensional cultured epidermis model (Tables 1 and 2). These data indicated that SPH is a stronger inhibitor of alkCDase than OEA. Subsequently, mice SC CER after application of SPH or OEA was determined. SPH concentration of $40 \mathrm{mM}$ was higher than the intracellular level. However, we considered that the SPH amount in the viable epidermis was low because SPH penetration was prevented by the SC barrier. CER in the SC was shown to be composed of 4 types of sphingoids; namely, SPH [S], dihydrosphingosine [DS], phytosphingosine $[\mathrm{P}]$, and 6-hydroxy sphingosine $[\mathrm{H}]$; and 3 types of FAs; namely, non-hydroxy FA [N], a-hydroxy FA [A], and esterified $\omega$-hydroxy FA [EO]. CER [AS] and CER [AP] were increased by application of SPH (Figure 3C and 3D). Mao et al. demonstrated that maCER1 ( $\mathrm{pH}$ 8.0) had specificity for CER containing D-erythrosphingosine, but CER had D-ribo-phytosphingosine and D-erythrodihydrosphingosine was not hydrolyzed [12]. Thus, it is likely that degradation of CER [AS], which contains D-erythro-sphingosine, was suppressed by inhibition of alkCDase. However, CER [NS] that also contained D-erythro-sphingosine, was not increased (Figure $3 \mathrm{~A})$; whereas, CER [AP] that contained D-ribo-phytosphingosine was increased (Figure 3D). Therefore, we considered that a mechanism other than alkCDase inhibition is also related to the increase of the CER. Further, the CER content in three-dimensional cultured epidermis model after application of SPH was also quantitated. We prepared a liposome containing SPH for application to three-dimensional cultured epidermis model because SPH was a lipophilic compound. The CER content of the control group with SPH-free liposome (DPPC/ DPPG, 4:1) was unchanged (Figure 6). Unlike the results on mice SC, CER [AS] and CER [AP] were unchanged; however, CER [NDS] was increased (Figure 6C-6E). Density of CER [NDS] in HPTLC analysis
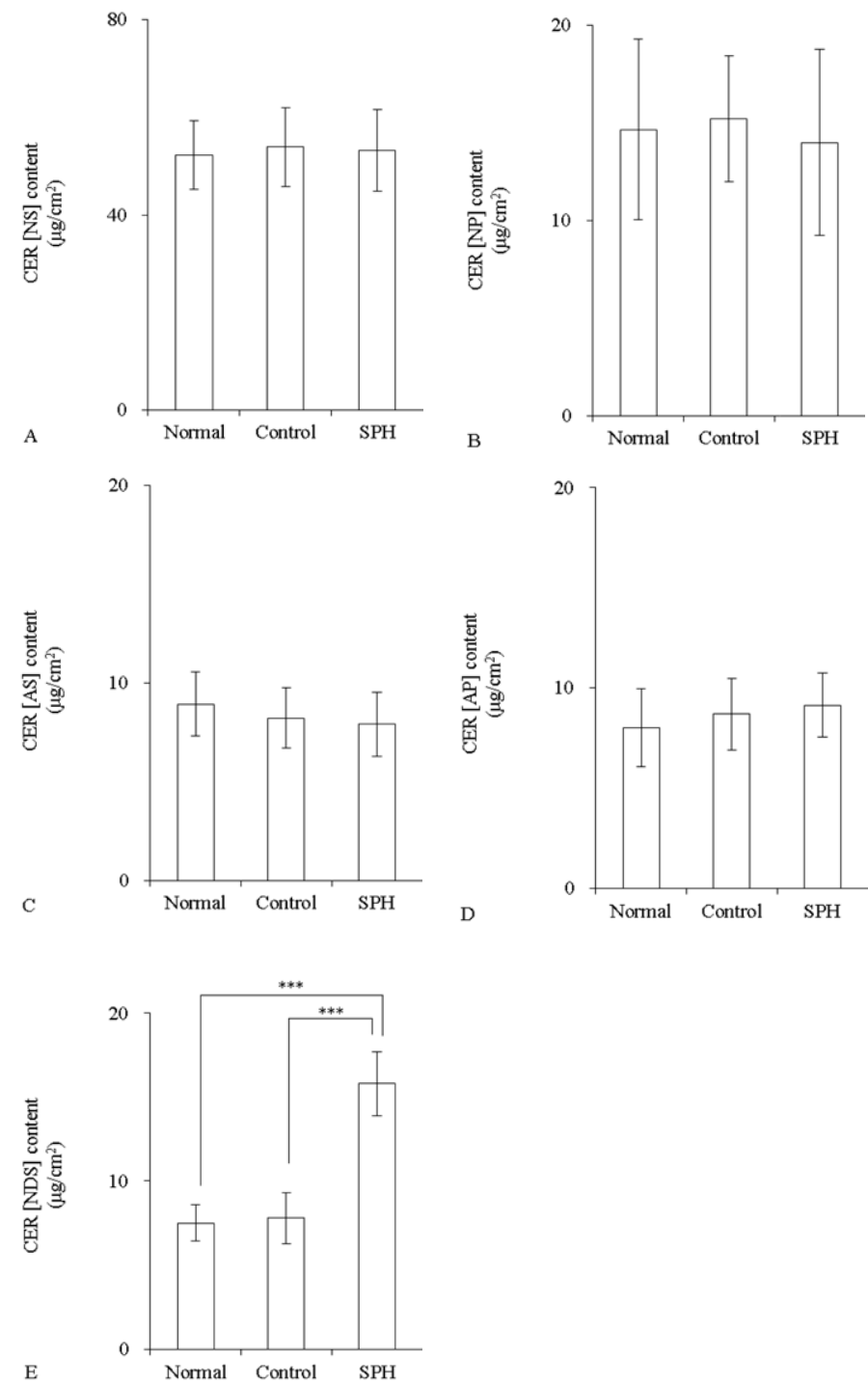

Figure 6: The CER content in three-dimensional cultured epidermis model after application of SPH liposome $(n=4)$

Liposome contained $10 \mathrm{mM}$ SPH. Control group was applied with SPH-free liposome (DPPC/DPPG, 4:1). Content of CER [NS] (A), CER [NP] (B), CER [AS] (C), CER [AP] (D), and CER [NDS] (E). Values are expressed as the mean \pm S.D. $* * * p<0.001$, determined by Turkey's test. 
from mice SC was unchanged (data not shown). CER [NDS] density was increased, in spite of no significant, by application of liposome containing $1 \mathrm{mM}$ SPH (data not shown). It was reported that CER [NP], CER $[\mathrm{NH}]$, and CER $[\mathrm{AH}]$ were popular classes $(22.1,14.5$, and $10.8 \%$ respectively) among the total CER content of human SC and that CER [EOS] accounted for 6.5\% [15]. Simotoyodome et al. also showed that CER [NP] and CER [NH] existed in abundance in human SC CER [16]. However, Liou et al. reported that CER [EOS] was dominant (approximately 75\%) in nude mice skin CER [17]. These reports indicated that the generation profile of individual SC CER classes maybe different between human and mice. Hence, we thought that different CER classes were increased between mice SC and threedimensional cultured epidermis model by the same compound. Duan et al. reported that the mRNAs of ceramide synthase (CerS) 2, CerS3, and CerS4 in normal human foreskin keratinocytes were upregulated by dihydrosphingosine, sphingosine, and 4,8-sphingadienine purified from maize glucosylceramide [18]. Shirakura et al. reported an increased mRNA expression of the enzyme related to CER production and that CER [EOS], CER [NS], and CER [NP] were increased by application of 4,8-sphingadienine and 4-hydroxy-8-sphinganine isolated from konjac glucosylceramide to three-dimensional cultured epidermis model [19]. Sigruener et al. also reported that sphingoids increased the mRNA level of sphingolipid metabolizes enzymes and the CER contents in human keratinocyte [20]. Thus, it is probable that de novo synthesis of CER was stimulated by SPH in our experiment. Sphingosine-1-phosphate (S1P) is generated from SPH by sphingosine kinase (SPHK) and induces keratinocyte differentiation [21]. Hong et al. demonstrated that specific differentiation-associated maker proteins in intact mice epidermis and $\mathrm{HaCaT}$ cells were increased by application of SPHK activator [22]. Thus, it is likely that SPH applied to mice or three-dimensional cultured epidermis model was metabolized to S1P and differentiation of keratinocyte was promoted; consequently, the CER was increased. It was known that a salvage pathway reconverted SPH to CER [23], but it was still unclear whether it contributed to skin barrier.

\section{Conclusion}

We revealed that SPH was a stronger inhibitor than OEA to increase the CER contents, indicating that SPH suppresses degradation of CER in SC by inhibition of alkCDase. However, SPH may also be affected by another pathway, like de novo synthesis. Thus, SPH may be a beneficial compound to increase skin CER.

\section{References}

1. Houben E, Uchida Y, Nieuwenhuizen WF, De Paepe K, Vanhaecke T, et al. (2007) Kinetic characteristics of acidic and alkaline ceramidase in human epidermis. Skin Pharmacol Physiol 20: 187-194. [Crossref]

2. Lin TK, Crumrine D, Ackerman LD, Santiago JL, Roelandt T, et al. (2012) Cellular changes that accompany shedding of human corneocytes. J Invest Dermatol 132: 24302439. [Crossref]

3. Houben E, Holleran WM, Yaginuma T, Mao C, Obeid LM, et al. (2006) Differentiationassociated expression of ceramidase isoforms in cultured keratinocytes and epidermis. J Lipid Res 47: 1063-1070. [Crossref]

4. Imokawa G, Abe A, Jin K, Higaki Y, Kawashima M, et al. (1991) Decreased level of ceramides in stratum corneum of atopic dermatitis: an etiologic factor in atopic dry skin? J Invest Dermatol 96: 523-526. [Crossref]

5. Ghadially R, Brown BE, Sequeira-Martin SM, Feingold KR, Elias PM (1995) The aged epidermal permeability barrier. Structural, functional, and lipid biochemical abnormalities in humans and a senescent murine model. J Clin Invest 95: 2281-2290. [Crossref]

6. Jin K, Higaki Y, Takagi Y, Higuchi K, Yada Y, et al. (1994) Analysis of beta- glucocerebrosidase and ceramidase activities in atopic and aged dry skin. Acta Derm Venereol 74: 337-340. [Crossref]

7. Higuchi K, Hara J, Okamoto R, Kawashima M, Imokawa G (2000) The skin of atopic dermatitis patients contains a novel enzyme, glucosylceramide sphingomyelin deacylase, which cleaves the $\mathrm{N}$-acyl linkage of sphingomyelin and glucosylceramide. Biochem J 350: 747-756. [Crossref]

8. Okino N, Tani M, Imayama S, Ito M (1998) Purification and characterization of a novel ceramidase from Pseudomonas aeruginosa. J Biol Chem 273: 14368-14373. [Crossref]

9. Okino N, Ichinose S, Omori A, Imayama S, Nakamura T, et al. (1999) Molecular cloning, sequencing, and expression of the gene encoding alkaline ceramidase from Pseudomonas aeruginosa. Cloning of a ceramidase homologue from Mycobacterium tuberculosis. J Biol Chem 274: 36616-36622. [Crossref]

10. Ohnishi Y, Okino N, Ito M, Imayama S (1999) Ceramidase activity in bacterial skin flora as a possible cause of ceramide deficiency in atopic dermatitis. Clin Diagn Lab Immunol 6: 101-104. [Crossref]

11. Yada Y, Higuchi K, Imokawa G (1995) Purification and biochemical characterization of membrane-bound epidermal ceramidases from guinea pig skin. J Biol Chem 270: 12677-12684. [Crossref]

12. Mao C, Xu R, Szulc ZM, Bielawski J, Becker KP, et al. (2003) Cloning and characterization of a mouse endoplasmic reticulum alkaline ceramidase: an enzyme that preferentially regulates metabolism of very long chain ceramides. $J$ Biol Chem 278: 31184-31191. [Crossref]

13. Houben E, Hachem JP, De Paepe K, Rogiers V (2008) Epidermal ceramidase activity regulates epidermal desquamation via stratum corneum acidification. Skin Pharmacol Physiol 21: 111-118. [Crossref]

14. Tokudome Y, Endo M, Hashimoto F (2014) Application of glucosylceramide-based liposomes increased the ceramide content in a three-dimensional cultured skin epidermis. Skin Pharmacol Physiol 27: 18-24. [Crossref]

15. t'Kindt R, Jorge L, Dumont E, Couturon P, David F, et al. (2012) Profiling and characterizing skin ceramides using reversed-phase liquid chromatography-quadrupole time-of-flight mass spectrometry. Anal Chem 84: 403-411. [Crossref]

16. Shimotoyodome Y, Tsujimura H, Ishikawa J, Fujimura T, Kitahara T (2014) Variations of ceramide profile in different regions of the body of Japanese females. Nippon Koshohin Gakkaishi 38: 3-8.

17. Liou YB, Sheu MT, Liu DZ, Lin SY, Ho HO (2010) Quantitation of ceramides in nude mouse skin by normal-phase liquid chromatography and atmospheric pressure chemical ionization mass spectrometry. Anal Biochem 401: 107-113. [Crossref]

18. Duan J, Sugawara T, Hirose M, Aida K, Sakai S, et al. (2012) Dietary sphingolipids improve skin barrier functions via the upregulation of ceramide synthases in the epidermis. Exp Dermatol 21: 448-452. [Crossref]

19. Shirakura Y, Kikuchi K, Matsumura K, Mukai K, Mitsutake S, et al. (2012) 4,8-Sphingadienine and 4-hydroxy-8-sphingenine activate ceramide production in the skin. Lipids Health Dis 11: 108. [Crossref]

20. Sigruener A, Tarabin V, Paragh G, Liebisch G, Koehler T, et al. (2013) Effects of sphingoid bases on the sphingolipidome in early keratinocyte differentiation. Exp Dermatol 22: 677-679. [Crossref]

21. Herzinger T, Kleuser B, Schäfer-Korting M, Korting HC (2007) Sphingosine-1phosphate signaling and the skin. Am J Clin Dermatol 8: 329-336. [Crossref]

22. Hong JH, Youm JK, Kwon MJ, Park BD, Lee YM, et al. (2008) K6PC-5, a direct activator of sphingosine kinase 1, promotes epidermal differentiation through intracellular $\mathrm{Ca}^{2+}$ signaling. J Invest Dermatol 128: 2166-2178. [Crossref]

23. Kitatani K, Idkowiak-Baldys J, Hannun YA (2008) The sphingolipid salvage pathway in ceramide metabolism and signaling. Cell Signal 20: 1010-1018. [Crossref]

Copyright: (C)2015 Nomoto K. This is an open-access article distributed under the terms of the Creative Commons Attribution License, which permits unrestricted use, distribution, and reproduction in any medium, provided the original author and source are credited. 